\title{
Correction to: Is There Enough Evidence for Osteosarcopenic Obesity as a Distinct Entity? A Critical Literature Review
}

\author{
Jürgen M. Bauer ${ }^{1}$ - Alfonso J. Cruz-Jentoft ${ }^{2} \cdot$ Roger A. Fielding $^{3}$ • John A. Kanis ${ }^{4,5}$ - Jean-Yves Reginster ${ }^{6,7,8}$. \\ Olivier Bruyère ${ }^{6,7} \cdot$ Matteo Cesari $^{9} \cdot$ Roland Chapurlat $^{10} \cdot$ Nasser Al-Daghri $^{8} \cdot$ Elaine Dennison $^{11}$. \\ Jean-Marc Kaufman ${ }^{12}$. Francesco Landi ${ }^{13}$. Andrea Laslop ${ }^{14}$. Médéa Locquet ${ }^{6,7}$. Stefania Maggi ${ }^{15}$. \\ Eugene McCloskey ${ }^{16,17}$. Simone Perna ${ }^{18} \cdot$ René Rizzoli $^{7,19}$. Yves Rolland ${ }^{20} \cdot$ Mariangela Rondanelli $^{21} \cdot$ Pawel Szulc $^{22}$. \\ Bruno Vellas $^{20} \cdot$ Mila Vlaskovska $^{23} \cdot$ Cyrus Cooper $7,11,24$
}

Published online: 23 July 2019

(c) The Author(s) 2019

\section{Correction to: Calcified Tissue International \\ (2019) 105:109-124 \\ https://doi.org/10.1007/s00223-019-00561-w}

The original version of this article unfortunately contained a mistake in one of the co-author's name. The coauthor Cyrus Cooper's degree "FMedSci" was incorrectly tagged as family name. This has been corrected with this erratum.

The original article has been corrected.
Open Access This article is distributed under the terms of the Creative Commons Attribution 4.0 International License (http://creativeco mmons.org/licenses/by/4.0/), which permits unrestricted use, distribution, and reproduction in any medium, provided you give appropriate credit to the original author(s) and the source, provide a link to the Creative Commons license, and indicate if changes were made.

Publisher's Note Springer Nature remains neutral with regard to jurisdictional claims in published maps and institutional affiliations.
The original article can be found online at https://doi.org/10.1007/ s00223-019-00561-w.

Jürgen M. Bauer

Juergen.Bauer@bethanien-heidelberg.de

Extended author information available on the last page of the article 


\section{Affiliations}

Jürgen M. Bauer ${ }^{1}$ - Alfonso J. Cruz-Jentoft ${ }^{2} \cdot$ Roger A. Fielding $^{3} \cdot$ John A. Kanis ${ }^{4,5}$ - Jean-Yves Reginster ${ }^{6,7,8}$. Olivier Bruyère $^{6,7} \cdot$ Matteo Cesari $^{9} \cdot$ Roland Chapurlat $^{10} \cdot$ Nasser Al-Daghri $^{8} \cdot$ Elaine Dennison $^{11}$. Jean-Marc Kaufman ${ }^{12}$. Francesco Landi ${ }^{13}$. Andrea Laslop ${ }^{14}$. Médéa Locquet ${ }^{6,7}$. Stefania Maggi ${ }^{15}$. Eugene McCloskey ${ }^{16,17}$. Simone Perna ${ }^{18} \cdot$ René Rizzoli $^{7,19}$. Yves Rolland ${ }^{20}$ - Mariangela Rondanelli ${ }^{21}$ - Pawel Szulc ${ }^{22}$. Bruno Vellas $^{20} \cdot$ Mila Vlaskovska $^{23} \cdot$ Cyrus Cooper $^{7,11,24}$

1 Center for Geriatric Medicine, University of Heidelberg, AGAPLESION Bethanien Krankenhaus Heidelberg, Heidelberg, Germany

2 Servicio de Geriatría, Hospital Universitario Ramón y Cajal (IRYCIS), Madrid, Spain

3 Nutrition, Exercise Physiology, and Sarcopenia Laboratory, Jean Mayer USDA Human Nutrition Research Center on Aging, Tufts University, Boston, MA, USA

4 Mary McKillop Health Institute, Australian Catholic University, Melbourne, Australia

5 Centre for Metabolic Bone Diseases, University of Sheffield Medical School, Sheffield, UK

6 Department of Public Health, Epidemiology and Health Economics, University of Liège, CHU Sart Tilman B23, 4000 Liège, Belgium

7 WHO Collaborating Centre for Public Health Aspects of Musculoskeletal Health and Aging, Liège, Belgium

8 Chair for Biomarkers of Chronic Diseases, Biochemistry Department, College of Science, King Saud University, Riyadh, Kingdom of Saudi Arabia

9 Fondazione IRCCS Ca' Granda Ospedale Maggiore Policlinico, University of Milan, Milan, Italy

10 INSERM, UMR 1033, Université de Lyon, Hôpital E Herriot, 69437 Lyon Cedex 03, France

11 MRC Lifecourse Epidemiology Unit, University of Southampton, Southampton General Hospital, Southampton, UK

12 Department of Endocrinology, Ghent University Hospital, Ghent, Belgium
13 Department of Geriatrics, Neurosciences and Orthopaedics, Orthogeriatric Unit, Teaching Hospital "Agostino Gemelli", Catholic University of the Sacred Heart School of Medicine, Rome, Italy

14 Scientific Office, Federal Office for Safety in Health Care, Austrian Agency for Health and Food Safety, Vienna, Austria

15 Aging Program, National Research Council, Padua, Italy

16 Centre for Metabolic Bone Diseases, University of Sheffield, Sheffield, UK

17 Mellanby Centre for Bone Research and Centre for Integrated Research in Musculoskeletal Ageing, University of Sheffield, Sheffield, UK

18 Department of Biology, College of Science, University of Bahrain, Sakhir Campus, P.O. Box 32038, Zallaq, Bahrain

19 Division of Bone Diseases, Geneva University Hospitals and Faculty of Medicine, Geneva, Switzerland

20 CHU Toulouse, Médecine Gériatrie Gérontopôle, Cité de la Santé, 20 Rue du Pont Saint Pierre, Inserm 1027, 31059 Toulouse, France

21 IRCCS Mondino Foundation, Pavia, Department of Public Health, Experimental and Forensic Medicine, Unit of Human Nutrition, University of Pavia, Pavia, Italy

22 INSERM, UMR 1033, University of Lyon, Hôpital Edouard Herriot, Lyon, France

23 Medical Faculty, Department of Pharmacology, Medical University Sofia, 2, Zdrave Str, 1431 Sofia, Bulgaria

24 NIHR Musculoskeletal Biomedical Research Unit, University of Oxford, Oxford, UK 